\title{
New Approach on the Techniques of Content-Based Image Retrieval (CBIR) Using Color, Texture and Shape Features
}

\author{
Mohd Afizi Mohd Shukran*, Muhamad Naim Abdullah, Mohd Sidek Fadhil Mohd Yunus \\ Department of Computer Science, Faculty of Defense Science and Technology, Universiti Pertahanan Nasional Malaysia, \\ Malaysia \\ Email: *afizi@upnm.edu.my
}

How to cite this paper: Shukran, M.A.M., Abdullah, M.N. and Yunus, M.S.F.M. (2021) New Approach on the Techniques of Content-Based Image Retrieval (CBIR) Using Color, Texture and Shape Features. Journal of Materials Science and Chemical Engineering, 9, 51-57.

https://doi.org/10.4236/msce.2021.91005

Received: December 29, 2020

Accepted: January 26, 2021

Published: January 29, 2021

\begin{abstract}
Digital image collection as rapidly increased along with the development of computer network. Image retrieval system was developed purposely to provide an efficient tool for a set of images from a collection of images in the database that matches the user's requirements in similarity evaluations such as image content similarity, edge, and color similarity. Retrieving images based on the content which is color, texture, and shape is called content based image retrieval (CBIR). The content is actually the feature of an image and these features are extracted and used as the basis for a similarity check between images. The algorithms used to calculate the similarity between extracted features. There are two kinds of content based image retrieval which are general image retrieval and application specific image retrieval. For the general image retrieval, the goal of the query is to obtain images with the same object as the query. Such CBIR imitates web search engines for images rather than for text. For application specific, the purpose tries to match a query image to a collection of images of a specific type such as fingerprints image and x-ray. In this paper, the general architecture, various functional components, and techniques of CBIR system are discussed. CBIR techniques discussed in this paper are categorized as CBIR using color, CBIR using texture, and CBIR using shape features. This paper also describe about the comparison study about color features, texture features, shape features, and combined features (hybrid techniques) in terms of several parameters. The parameters are precision, recall and response time.
\end{abstract}

\section{Keywords}

Content-Based Image Retrieval, Image Retrieval, Information Retrieval 


\section{Introduction}

With the widespread use of computer network, collection of information data such as image has quickly grown and continues to increase in the future. Due to rapidly grown of internet, every image information and data was collected and digitized. Every internet user was able to access it because all that image information and data available in digital image format.

In order to access this huge amount of data, efficient technique and method is needed for querying the indexed image database. In order to find and locate the needed information from these large databases, it is difficult and surely will take a lot of time. So, a method called content-based image retrieval is the efficient retrieval method to find relevant and needed images. Content Based Image Retrieval (CBIR) system was introduced in 1990 [1]. It is basically a techniques used for automatic retrieval of images in a large database that perfectly matches the query image.

Basically, in content based image retrieval system, it consists of two kinds of content based image retrieval which are general image retrieval and application specific image retrieval. For the general image retrieval, the goal of the query is to obtain images with the same object as the query. Such CBIR imitates web search engines for images rather than for text. For application specific, the purpose tries to match a query image to a collection of images of a specific type such as fingerprints image and $\mathrm{x}$-ray. The need for efficient finding, searching and retrieval of digital image has been rapidly increase in many image processing application, viz, medicine, commerce, crime prevention, military, education, culture, and entertainment [2].

\section{CBIR Architectural Design}

Basically a general CBIR system architecture consists of six functional blocks which is image database (the block that contains image of the chosen database), feature database (can be characterized by a set of features), feature extraction (features may be text-based features and visual features), query image (can be any of the database image), image matching and indexing (the features of query image are compared with features that are already stored in the feature database), and retrieved image (the system that will select any number of image having the highest overall similarity to the given query image and present to the user as retrieved images[2]. Figure 1 shows the general architecture of CBIR system.

\section{CBIR Techniques}

Generally, in CBIR systems, while building an image database or retrieving an image from the database, feature vectors are the first extracted from images. The features can be color, texture, shape, and the combination of these features (hybrid features) [2]. These features are then used to build the feature database. When query image is given, its feature vectors are extracted and similarity matching 


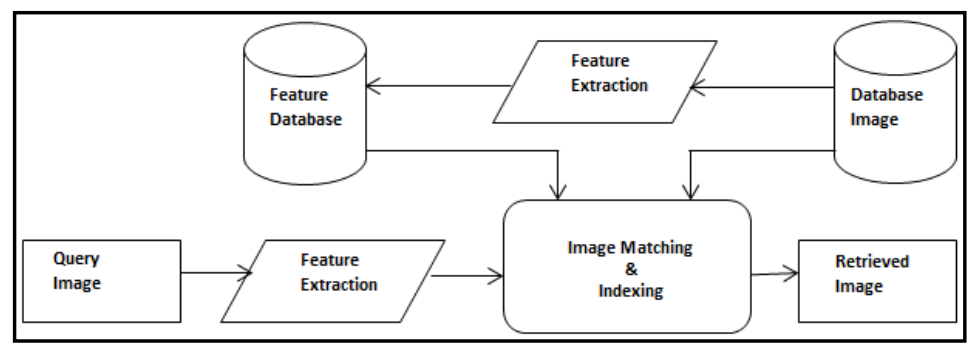

Figure 1. Content-based image retrieval system general architecture.

is performed with the feature vectors already present in feature database as illustrated in Figure 1 in previous section.

\subsection{CBIR Using Color Features}

Color is one of the important features that make possible the recognition of image by human. J. Yue et al. in 2011, state that the color is one of the most widely used low-level visual features and is invariant to image size and orientation [3]. According to B.S. Manjunath et al., color features are the most expressive of all the visual features and have been extensively used in the image retrieval system [4].

Swain and Ballard have proposed a color matching method based on color indexing in early 1990s. This method is also known as color histogram intersection method and it is based on matching of color histograms [2]. In 2011, G. H. Liu et al. state in his paper that, color histogram is invariant to orientation and scale and this makes it powerful in image classification. Subsequently, color histogram-based color descriptor has been broadly studied and generally utilized in CBIR systems for its simplicity and effectiveness [5].

\subsection{CBIR Using Texture Features}

Another important element in visual perception is "Texture". Texture also can be used to separate regions of interest in an image. According to Savita et al. in 2015, texture is also one of the most used low level visual features that refer to innate surface properties of an object and their relationship to the surrounding environment and it also contains important information about the structural arrangement of surfaces and their relationship with the surroundings [6].

According to M. Kokare et al., texture can be defined as, "a region in image has a constant texture if a set of local statistics or other local properties of the picture are constant, gradually differing or approximately periodic" [7]. In 1998, Y. S. Kim and W. Y. Kim explored that the methodology for existing texture classification can be roughly divided into three categories which are Statistical method, Structural method, and Model-based method [8].

Some of the algorithm for CBIR based on this texture as feature has been proposed by several researchers. In 2000s, a rotational invariant texture features using Dual-Tree Complex Wavelet Transform (DTCWT) for CBIR has been proposed by P. R. Hill, D. R. Bull, and C. N. Canagarajah, [9]. In 2003, C. M. 
Pun has proposed an effective rotation-invariant polar-wavelet texture feature for image retrieval [10]. Other than that, in 2005 M. Kakore et al. proposed texture feature for image retrieval that supports shift invariant by using a set of two-dimensional Dual Tree Complex Wavelet Transform (DTCWT) and Dual Tree Rotated Complex Wavelet Filters (DTRCWF) jointly [11]. J. Han and K. K. $\mathrm{Ma}$ in 2007 has presented texture features for image retrieval using Gabor wavelet. In their proposal, mean and standard deviation values of transformed coefficients computed individually found to be rotation and scale invariant [12]. In 2009, a texture image retrieval methodology based on non-tensor product wavelet filter banks has been proposed by Z. He, X. You, and Y. Yuan [13].

\subsection{CBIR Using Shape Features}

In content-based image retrieval system, another important visual feature is shape. According to D. Zhang and G. Lu in 2004, shape is one of the basic features used to describe image content and it is also can be used to provide powerful information. Nevertheless, shape representation and description is a difficult task because when a 3-D real world object is projected onto a 2-D image plane, one dimension of object information is lost. As a result, the shape extracted from the image only partially represents the projected object. Further, shape is often corrupted with noise, defects, arbitrary distortion and occlusion [14].

According to Rao \& Kumar in 2012, shape representation and description methods can be divided into two main categories which is boundary-based and region-based [2]. Savita Gandhani et al. in 2015 stated that the boundary-based technique will use only the contour or border of the object and completely ignores its interior, whereas region-based technique apply segmentation to divide an image into different regions/segments, by setting threshold values according to the desirable results [6].

According to Sharmin Siddique, the most successful representations for shape categories are Fourier Descriptor and Moment Invariants. She stated in her paper, the main idea of Fourier Descriptor is to use the Fourier transformed boundary as the shape feature, whereas Moment invariants is to use region-based moments, which are invariant to transformations as the shape feature [15].

\subsection{Comparison Study for Color, Texture, Shape and Combined (Hybrid) Features}

In content-based image retrieval system, some key parameters have been defining and evaluate based on precision, recall and the response time. The image retrieval precision rate and recall rate can be defined as this following formula:

$$
\begin{aligned}
& \text { precision }=\frac{R}{N} \\
& \text { recall }=\frac{R}{M}
\end{aligned}
$$




\begin{tabular}{|l|c|c|c|}
\hline Method/Item & Precision & Recall & Response Time (ms) \\
\hline Texture Feature & $60.1 \%$ & $72.3 \%$ & 1373 \\
\hline Color Feature & $53.7 \%$ & $65.2 \%$ & 824 \\
\hline Shape Feature & $62.2 \%$ & $70.8 \%$ & 2544 \\
\hline Combined Feature & $79.6 \%$ & $88.3 \%$ & 3861 \\
\hline
\end{tabular}

Figure 2. Comparison study for color, texture, shape and combined (hybrid) features.

The $N$ is stand for the number of images returned for the query, whereas $R$ is images associated with the example in the result. Besides, $M$ is stand for images associated with the example in test set $\mathrm{S}$ (saturation) [16].

Based on comparison study by D. Guoyong et al. in 2011, for the texture feature technique, the precision percentage is $60.1 \%$, recall percentage score is $72.3 \%$, and response time is 1372 millisecond. Other than that, for the color feature technique, the precision percentage score is $53.7 \%$, recall percentage is $65.2 \%$, and response time is 824 milliseconds. Besides that, for the shape feature technique, the precision percentage is $62.2 \%$, recall percentage is $70.8 \%$, and response time is 2544 millisecond. Last but not least, for the combined feature technique, the precision percentage is $79.6 \%$, recall percentage is $88.3 \%$, and response time is 3861 milliseconds [16] (Figure 2).

Based on the comparison study, for the first parameter which is precision percentage, combined feature is the highest. For second parameter, which is recall percentage, combined feature is the highest. Besides, for the third parameter which is response time, color feature is the fastest. Based on that comparison study, the combination or hybrid features technique which is combination of texture, color and shape strongly improved precision and recall, although it has longer response time.

\section{Conclusion}

Image features play an important function in retrieving suitable images from the large image database. Effective image retrieval from database requires exploration of suitable features such as color, text and shape of images in the database. Appropriately, many previous researchers have presented and proposed several image retrieval algorithms by exploring various features and transforms. In this paper, various CBIR techniques and algorithms are briefly described and discussed. Based on the literature study, researcher also need to find a good combination of image feature with its own similarity measure because the major requirement of an ideal CBIR system is to develop computational faster CBIR algorithm with improved average retrieval efficiency. 


\section{Conflicts of Interest}

The authors declare no conflicts of interest regarding the publication of this paper.

\section{References}

[1] Shriwas, M.K. and Raut, V.R. (2015) Content Based Image Retrieval: A Past, Present and New Feature Descriptor. 2015 International Conference on Circuits, Power and Computing Technologies (ICCPCT-2015). https://doi.org/10.1109/ICCPCT.2015.7159404

[2] Rao, C.S. and Kumar, S.S. (2012) Content Based Image Retrieval Fundamentals \& Algorithms. LAP Lambert Academic Publishing.

[3] Yue, J., Li, Z., Liu, L. and Fu, Z.T. (2011) Content-Based Image Retrieval Using Color and Texture Fused Features. Mathematical and Computer Modelling, 54, 1121-1127. https://doi.org/10.1016/j.mcm.2010.11.044

[4] Manjunath, B.S., Ohm, J.R., Vasudevan, V.V. and Yamada, A. (2001) Color and Texture Descriptors. IEEE Transactions on Circuit and Systems for Video Technology, 11, 703-715.

[5] Liu, G.H., Li, Z.Y., Zhang, L. and Xu, Y. (2011) Image Retrieval Based on Micro-Structure Descriptor. Pattern Recognition, 44, 2123-2133A. https://doi.org/10.1016/j.patcog.2011.02.003

[6] Gandhani, S. and Singhal, N. (2015) Content Based Image Retrieval: Survey and Comparison of CBIR System Based on Combined Features. International Journal of Signal Processing, Image Processing and Pattern Recognition, 8, 155-162. https://doi.org/10.14257/ijsip.2015.8.10.18

[7] Kokare, M., Chatterji, B.N. and Biswas, P.K. (2002) A Survey on Current Content-Based Image Retrieval Methods. IETE Journal of Research, 4. 261-271. https://doi.org/10.1080/03772063.2002.11416285

[8] Kim, Y.S. and Kim, W.Y. (1998) Content-Based Trademark Retrieval System Using a Visually Salient Features. Image and Vision Computing, 16, 931-939. https://doi.org/10.1016/S0262-8856(98)00060-2

[9] Hill, P.R., Bull, D.R. and Canagarajah, C.N. (2000) Rotationally Invariant Texture Features Using the Dual-Tree Complex Wavelet Transform. Proceeding of IEEE International Conference on Image Processing, 3, 901-904.

[10] Pun, C.M. (2003) Rotation Invariant Texture Features for Image Retrieval. Computer Vision and Image Understanding, 89, 24-43. https://doi.org/10.1016/S1077-3142(03)00012-2

[11] Kokare, M., Biswas, P.K. and Chatterji, B.N. (2005) Texture Image Retrieval Using New Rotated Complex Wavelet Filters. IEEE Transaction on Systems, Man and Cybernatics, 35, 1168-1178. https://doi.org/10.1109/TSMCB.2005.850176

[12] Pan, Z., Zheng, Y., Zhang, R. and Jenkins, B.M. (2007) Physical Properties of Thin Particleboard Made from Saline Eucalyptus. Industrial Crops and Products, 26, 185-194. https://doi.org/10.1016/j.indcrop.2007.03.006

[13] He, Z., You, X. and Yuan, Y. (2009) Texture Image Retrieval Based on Non-Tensor Product Wavelet Filter Banks. Signal Processing, 89, 1501-1510. https://doi.org/10.1016/j.sigpro.2009.01.021

[14] Zhang, D. and Lu, G. (2004) Review of Shape Representation and Description Techniques. Pattern Recognition, 37, 1-19. 


\section{https://doi.org/10.1016/j.patcog.2003.07.008}

[15] Siddique, S. (2002) A Wavelet Based Technique for Analysis and Classification of Texture Images. Carleton University, Ottawa, Canada, Proj. Rep. 70.593.

[16] Duan, G.Y., Yang, J. and Yang, Y.L. (2011) Content Based Image Retrieval Research. 2011 International Conference on Physics Science and Technology (ICPST 2011), 471-477. https://doi.org/10.1016/j.phpro.2011.11.073 